\title{
Putative Loci Causing Early Embryonic Mortality in Duroc Swine
}

\author{
Chunyan Zhang ${ }^{1}$, Michael D. MacNeil ${ }^{2,3}$, Robert A. Kemp ${ }^{4}$, Michael K. Dyck ${ }^{1}$ and \\ Graham S. Plastow ${ }^{1 *}$
}

'Department of Agricultural, Food \& Nutritional Sciences, University of Alberta, Edmonton, AB, Canada, ${ }^{2}$ Delta G, Miles City, MT, United States, ${ }^{3}$ Department of Animal, Wildlife and Grassland Sciences, University of the Free State, Bloemfontein,

South Africa, ${ }^{4}$ Genesus Inc., Oakville, MB, Canada

Lethal recessive alleles that act prenatally may be detected from the absence of homozygous individuals in a population. However, these alleles may be maintained at relatively low frequencies in populations as heterozygotes. In pigs, they may reduce litter size. This study aimed to detect putative lethal variants in the Duroc breed. Phenotypes for the numbers of piglets born (TNB), born live (BA), alive at $24 \mathrm{~h}(\mathrm{~L} 24)$, stillborn (SB), and born as mummified fetuses (MM) were available from 5340 recorded litters which resulted from mating of 192 genotyped boars with sows of unknown genotype (dataset 1). An additional 50 litters were produced from parents that were both genotyped (dataset 2). Imputed genotypes of 650K SNPs for 1359 Duroc boars were used in this study. One significant SNP (Bonferroni corrected $P=5.5 \mathrm{E}-06$ ) was located on SSC14 with 45.3 homozygous individuals expected but none observed. This SNP was

OPEN ACCESS

Edited by:

Luis Varona,

Universidad de Zaragoza, Spain

Reviewed by:

Luca Fontanesi,

Università degli Studi di Bologna, Italy Fabyano Fonseca Silva,

Universidade Federal de Viçosa, Brazil

*Correspondence:

Graham S. Plastow plastow@ualberta.ca

Specialty section:

This article was submitted to Livestock Genomics,

a section of the journal

Frontiers in Genetics

Received: 24 April 2018 Accepted: 03 December 2018 Published: 17 December 2018

Citation:

Zhang C, MacNeil MD, Kemp RA Dyck MK and Plastow GS (2018)

Putative Loci Causing Early Embryonic Mortality in Duroc Swine. Front. Genet. 9:655.

doi: 10.3389/fgene.2018.00655 significant for mummified fetuses. One hundred fifty two haplotypes were also found to potentially harbor recessive lethal mutations. Twenty-one haplotypes had a significant harmful effect on at least one trait. Two regions, located on SSC8 (144.9-145.5 Mb) and SSC9 (19-19.4 Mb) had significant effects on fertility traits in both datasets. Additionally, regions on SSC1 (82.0-82.8 Mb), SSC3 (73.3-73.7 and 87.1-87.5 Mb) and SSC12 (35.8-36.2 and 50.0-50.5 Mb) had significant deleterious effects on TNB or BA or L24 in dataset 1. Finally, a region on SSC17 (28.7-29.3 Mb) had significant effects on TNB, BA and L24 in dataset 2. A few candidate genes identified within these regions were described as being involved in spermatogenesis and male fertility (TEX14, SEP4, and HSF5), or displayed recessive lethality (CYP26B1, SCD5, and PCF11) in other species. The putative loci detected in this study provide valuable information to potentially increase Duroc litter size by avoiding carrier-by-carrier matings in breeding programs. Further study of the identified candidate genes responsible for such lethal effects may lead to new insights into functions regulating pig fertility.

Keywords: Duroc pigs, lethal recessive loci, fertility, haplotype blocks, 650K SNP chip

\section{INTRODUCTION}

Comparatively small litters are characteristic of the Duroc breed (Johnson, 1981; Sonderman and Luebbe, 2008). Yet, use of Duroc as a terminal sire is a mainstay of swine production due to favorable direct effects on growth and carcass traits (Johnson, 1981; Zhang et al., 2016). Early embryonic mortality, affecting litter size, is recognized as a significant issue in swine (Bolet, 1986; Christianson, 1992). Geisert and Schmitt (2002) noted a major limitation for increasing litter size 
in swine is embryonic loss that occurs during the second to third week of gestation. Many factors may contribute to this loss and most work has focused on increasing litter size in the maternal lines based on Landrace and Large White breeds where high ovulation rates exist and litter size is relatively high and responds to selection (Sorensen et al., 2000; Noguera et al., 2002). However, little is known about ovulation rate in Duroc sire lines and there has been little selection for increased litter size in this breed as the focus of genetic improvement programs has been on performance and carcass quality (Lonergan et al., 2001). One possible cause of low litter size in the Duroc may be the existence of recessive lethal mutations which result in embryonic loss. With the generation of large amounts of genomic data this hypothesis can now be tested where loci harboring lethal recessive alleles may be discovered from the absence of live animals that are homozygous. Precedent for this proposed mechanism is found in recent studies based on observations of missing homozygous haplotypes in cattle (VanRaden et al., 2011; Hoff et al., 2017; Howard et al., 2017). Only genotypes from apparently normal individuals and not from affected embryos are required. Thus, under the assumptions that neither ovulation rate (Young et al., 1976) nor uterine capacity prior to fetal implantation are limiting in Duroc, we hypothesized that early embryonic mortality due to homozygosity of recessive lethal alleles may contribute to the production of relatively small litters by Duroc females. Due to the low reproductive rate of Duroc as a purebred there is: (1) an immediate cost due to a reduction in number of pigs produced per litter; and (2) a recurrent cost due to a decreased rate of genetic improvement resulting from reduced selection intensity. Therefore, the primary objective of this research was to scan the Duroc genome for segregating individual single nucleotide polymorphisms (SNPs) and haplotypes for which one homozygous genotype is either absent or in unexpectedly low frequency. A secondary objective was to test the association of any candidate loci with phenotypes (total number of born, number of born alive, number alive at $24 \mathrm{~h}$, stillborn piglets, and number of mummified fetuses) that are related to fertility of swine. The third objective was to identify some candidate genes that might be involved in functional pathways of sow fertility.

\section{MATERIALS AND METHODS}

DNA from 1359 Duroc boars born between 2012 and 2013 was genotyped using the Geneseek-Neogen GPPHD 80K SNP chip $^{1}$. The pedigree of these boars was used to identify 171 of their sires and grandsires. DNA from these ancestors was genotyped using the Affymetrix Axiom ${ }^{\circledR}$ 650K SNP Array. DNA extraction and genotyping were conducted the Delta Genomics laboratory (Edmonton, AB, Canada). The genotypes and details of genotyping and imputation were described by Zhang et al. (2018). Briefly, average imputation accuracy (percentage of correctly imputed SNPs) from 80 to $650 \mathrm{~K}$ was $92.1 \%$ and only those SNPs with accuracy of imputation $\geq 95 \%$ were used in this study. Additional quality control standards included: animal call

${ }^{1}$ http://bioinformatics.tecnoparco.org/SNPchimp/index.php/data-source rate $\geq 95 \%$, SNP call rate $\geq 95 \%$, SNPs mapped to the autosomal chromosomes (Sscrofa10.2), minor allele frequency (MAF) $\geq 5 \%$, and Chi square of Hardy-Weinberg equilibrium test less than 600. After editing, a total of 416,392 SNPs were used for this study.

\section{Detection of an Absent Homozygous Genotype}

For each SNP, the expected number of animals with a homozygous genotype was calculated assuming random mating and determined as the number of genotyped animals multiplied by the square of the MAF. Those SNPs with greater than five individuals expected to be homozygous for the minor allele, but where none were observed in the data, were considered to be necessary for life. Further, the difference between the observed and expected number of homozygotes was tested using a Chisquared test $(\mathrm{df}=1)$. The Bonferroni-corrected significance threshold was set as $P<0.01$.

Genotypes were phased on each chromosome using SHAPEIT2 (Delaneau et al., 2012, 2014) with an average window size of $2 \mathrm{Mb}$ and effective population size of 75 as recently reported for Canadian Duroc by Grossi et al. (2017). As there is no complete genetic map for pigs, a consistent recombination rate was given for each chromosome according to the average value from Tortereau et al. (2012). The phased genotypes were used to construct haplotype blocks and detect blocks containing potential recessive lethal alleles using GHap (Utsunomiya et al., 2016). The optimal window size was defined as $400 \mathrm{~Kb}$ with a sliding block of $100 \mathrm{~Kb}$, following Veroneze et al. (2013), in which it was reported that the average extent of linkage disequilibrium (LD) in pig was about $400 \mathrm{~Kb}$.

The expected number of animals with a homozygous haplotype within each window was calculated assuming random mating as no direct selection for litter size occurred in this population and determined as the number of genotyped animals divided by 4 and multiplied by the square of the carrier frequency of that particular haplotype (VanRaden et al., 2011). The blocks with 3 SNPs or less, or haplotype alleles with MAF $<0.01$ were excluded. The difference between the observed and expected number of homozygotes was again tested by Chi-squared of Hardy-Weinberg equilibrium distribution. The $P$-value from the Chi-square test was adjusted for whole genome wide significance by Bonferroni correction. A haplotype allele was defined as a putative recessive lethal if the observed number of homozygotes was zero and the expected number of homozygotes was greater than 6 as suggested by previous studies (Sahana et al., 2013; Haggman and Uimari, 2017), and a Bonferroni corrected $P$-value $<0.01$.

\section{Association Analysis}

Between 2006 and 2016, 192 genotyped boars sired 5340 recorded litters from the sows with unknown genotypes (dataset 1). Five traits: numbers born (TNB), born alive (BA), alive at $24 \mathrm{~h}$ (L24), stillborn piglets (SB), and mummified fetuses (MM) were used in association analyses 
to evaluate the effect of the detected haplotypes on fertility. Although they were count data, the distributions of TNB, BA and L24 were approximately normal and they were analyzed in linear animal models in ASREML (Gilmour et al., 2008). The distributions of SB and MM were Poisson and a Poisson regression animal model in the MCMCglmm R package (Hadfield, 2010) was used for their association analyses. The model was described as below.

$$
y=X b+M i+Z a+W v+e
$$

In this model, $y$ is the observed phenotype, $\boldsymbol{X}$ is an incidence matrix associated with fixed effects of parity $(1,2,3,4$, $5,>6$ ) (not included for $\mathrm{MM}$ ) and farrowing year-season (37 levels), $\boldsymbol{b}$ is the vector of those fixed effects, $\boldsymbol{M}$ is the design matrix of haplotype alleles $(0=$ non-carrier, 1 = carrier), $i$ is the vector of corresponding haplotype allele effect on observation (linear regression) or log of observation (Poisson regression), $\boldsymbol{Z}$ is an incidence matrix indicating random additive genetic effects $(a)$ due to boars with mean 0 and (co)variance structure of $A \sigma_{\mathrm{a}}^{2}$ where $\boldsymbol{A}$ is the pedigree-based additive relationship matrix and $\sigma_{a}^{2}$ is the additive genetic variance, $W$ is an incidence matrix indicating random effects $(v)$ due to sows with (co)variance structure of $I \sigma_{\mathrm{e}}^{2}$ where $I$ is identical matrix and $\sigma_{\mathrm{e}}^{2}$ is sow random variance, and $\boldsymbol{e}$ is a vector of random residual errors.
Additionally, there were 50 litters with known mating types which were generated from 27 genotyped boars mated with 22 genotyped sows (dataset 2). This information was suitable to estimate the effect of the lethal haplotype combination on TNB, BA an L24, but not for SB or MM due to small numbers of observations. Mating type for each detected lethal haplotype allele, including carrier mated with carrier (11), non-carrier mated with non-carrier (00) and carrier mated with non-carrier (10 or 01$)$, was fitted as a fixed effect in the above linear model $\left(\boldsymbol{M}_{\mathrm{i}}\right)$ to test their effect and significance. Due to the small sample size, the population mean was the only fixed effect that was included in the model. The significant threshold was set as $P<0.10$.

\section{Candidate Gene Identification}

Genes located within or flanking a detected significant haplotype block by less than $400 \mathrm{~Kb}$ were identified using pig genome assembly of Sscrofa 10.2. ${ }^{2}$ Initial identification of candidate genes was based on the annotated functions and related information that has been summarized in the GENECARDS ${ }^{3}$ database. Only the genes which were related to fertility functional pathways were reported and discussed in this paper.

\footnotetext{
${ }^{2}$ http://www.ensembl.org/biomart/martview

${ }^{3}$ http://www.genecards.org/
}

TABLE 1 | Location and minor allele frequency for haplotypes which were expected to have two homozygous genotypes, but with only one found in the data and which had a significant effect on at least one of the fertility phenotypes.

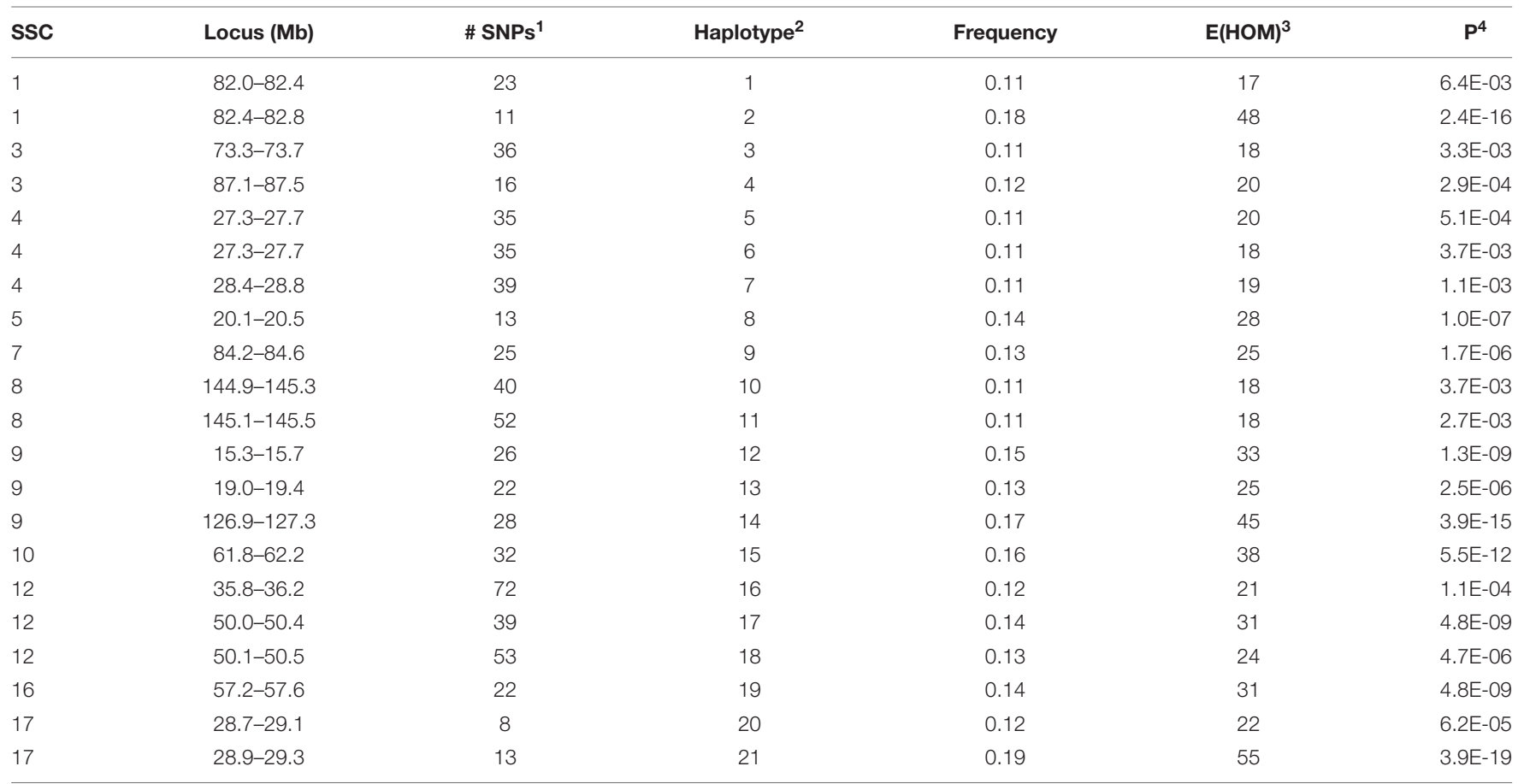

${ }^{1}$ Number of SNPS in the haplotype blocks; ${ }^{2}$ Haplotype index; ${ }^{3}$ number of individuals that were expected to be homozygous for the minor allele; ${ }^{4}$ Bonferroni corrected P-value. 


\section{RESULTS AND DISCUSSION}

\section{Single SNP and Haplotype Detection}

Only one SNP was found to have a sufficient expected number of homozygous recessive individuals with none being observed to attain statistical significance (Bonferroni corrected $P=5.5 \mathrm{E}$ 06). It had a MAF of 0.17 and thus it was expected that 45.3 homozygous recessive individuals would have been found. This SNP showed an effect on MM that litters sired by the boars carrying such allele had an average of 0.15 more log of mummified fetuses than the litters sired by non-carrier boars $(P=0.09)$. This SNP was on SSC14 at $59.5 \mathrm{Mb}$. Howard et al. (2017) observed a haplotype at $60.26-60.62 \mathrm{Mb}$ on SSC 14 that was significantly associated with TNB in Large White.

A total of 152 haplotypes from 129 blocks were detected as potentially harboring recessive lethal mutations with Bonferronicorrected $P<0.01$. The SNP on SSC14 identified above was contained within one of these haplotypes. Twenty-one

TABLE 2 | Effect $(P<0.10)$ of sire haplotype (non-carrier $=0$, carrier $=1$ ) on numbers of piglets born (TNB), born live (BA) and alive at $24 \mathrm{~h}$ postpartum (L24) as expressed in litters of purebred Duroc pigs.

\begin{tabular}{lccccc}
\hline Haplotype & SSC & Locus (Mb) & Trait $^{1}$ & \multicolumn{2}{c}{ Sire haplotype } \\
\cline { 5 - 6 } & & & & $\mathbf{0}$ & $\mathbf{1}$ \\
\hline 2 & & & & & \\
3 & & & & & \\
3 & 3 & $73.4-82.8$ & TNB & $10.51 \pm 0.17$ & $10.32 \pm 0.18$ \\
4 & 3 & $87.1-87.5$ & TNB & $10.49 \pm 0.17$ & $10.18 \pm 0.20$ \\
& & & BA & $9.32 \pm 0.19$ & $9.08 \pm 0.22$ \\
10 & 8 & $144.9-145.3$ & TNB & $10.51 \pm 0.18$ & $10.28 \pm 0.19$ \\
11 & 8 & $145.1-145.5$ & TNB & $10.53 \pm 0.18$ & $10.25 \pm 0.19$ \\
& & & L24 & $9.11 \pm 0.17$ & $8.82 \pm 0.19$ \\
12 & 9 & $15.3-15.7$ & L24 & $9.08 \pm 0.17$ & $8.88 \pm 0.18$ \\
13 & 9 & $19-19.4$ & TNB & $10.53 \pm 0.18$ & $10.24 \pm 0.19$ \\
& & & BA & $9.36 \pm 0.19$ & $9.11 \pm 0.21$ \\
16 & 12 & $35.8-36.2$ & L24 & $9.05 \pm 0.17$ & $8.82 \pm 0.20$ \\
18 & 12 & $50.1-50.5$ & TNB & $10.53 \pm 0.18$ & $10.25 \pm 0.19$
\end{tabular}

of these haplotypes also had significant effects on at least one fertility trait (see below) and were presented in Table 1. There were 131 additional haplotypes that were deficient of a homozygous genotype for the minor allele (Supplementary Table S1). However, none of these latter haplotypes had a detectable effect on any of the fertility traits. These haplotypes are located on all autosomes except for SSC13 and SSC18. Each haplotype contained, on average, 32.6 (5 - 85) SNPs. The carrier frequency for these haplotypes ranged from 0.10 to 0.26 and the expected number of homozygous individuals varied from 17 to 102.

A few recent studies report potential harmful recessive haplotypes in pigs. Haggman and Uimari (2017) detected 26 putative lethal haplotypes in Finnish Yorkshire AI boars, with the most important one was located on SSC8 at 107.0 - 113.3 Mb which was significantly associated with number of stillborn piglets in first and later parities. In this study we detected three regions on SSC8 although they were all distant to the region detected in the Finnish Yorkshire AI boars. These regions included 144.9-145.5 Mb (Haplotypes 10-11), 28.3-28.7 Mb (Haplotype 80) and 49.549.9 Mb (Haplotype 81). The first region (Haplotypes 10-11) showed a significant effect on TNB and L24 which will be discussed below. Two other regions detected in this study, 46.5 - 47.3 Mb on SSC4 (Haplotypes 52-54) and 110.1 110.6 Mb on SSC15 (Haplotypes 120-122), were close to regions detected by Haggman and Uimari (2017) at SSC4: 48.33 - 50.61 Mb and SSC15: 111.32 - $113.19 \mathrm{Mb}$. A different study by Howard et al. (2017) found six regions containing putative lethal recessive haplotypes that significantly affected TNB in Large White pigs. Three of them were the same or very close to the regions we detected here, including SSC1: $137 \mathrm{Mb}$, SSC10: $29 \mathrm{Mb}$, and SSC14: $60 \mathrm{Mb}$. In our study, the latter region on SSC14 contained the most significant SNP (14_59487297) detected by single SNP analyses. A third study by Derks et al. (2017) detected 145 haplotypes and 35 of them showed a negative effect on at least one of the analyzed reproductive traits. The region on SSC14 at 146 $150 \mathrm{Mb}$ was also detected in our study (Supplementary Table S1) although it was not found to have an impact on

TABLE 3 | Estimated difference between sire haplotypes (non-carrier - carrier) on numbers of stillborn piglets (SB) and mummified fetuses (MM) as expressed in litters of purebred Duroc pigs.

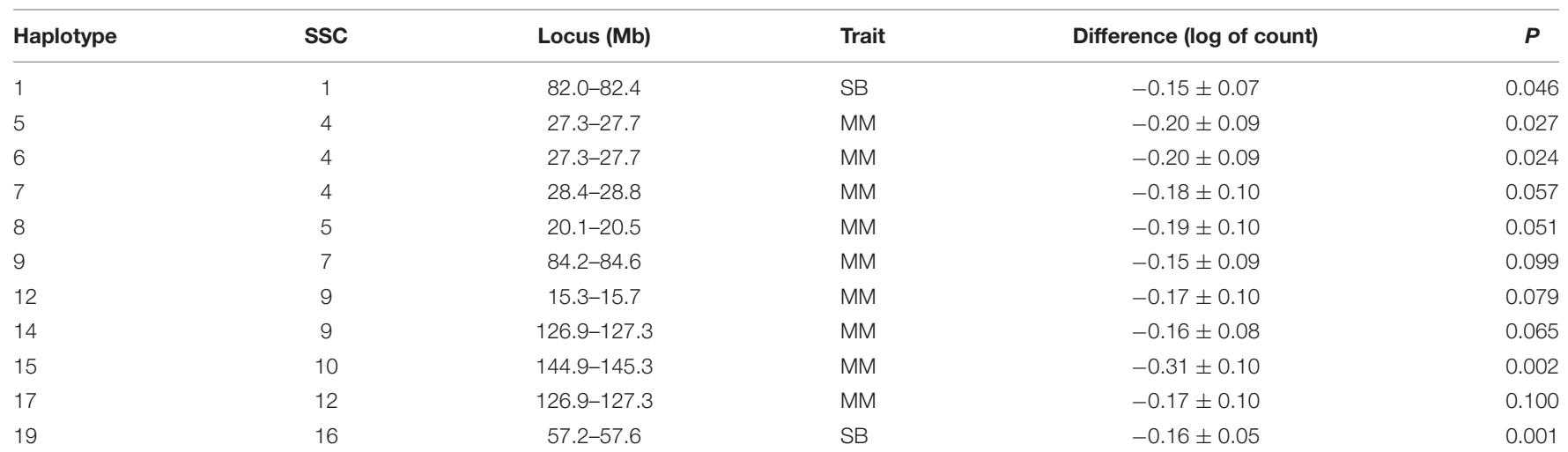


fertility. Other important regions detected in our study include SSC4:74.7 - 75.1 Mb (Haplotype 56), SSC6:111 Mb (Haplotypes 66-68), SSC9:127 Mb (Haplotypes 84-85) and SSC12:36Mb (Haplotype 16, Haplotypes 97-98), which were reported as harboring QTL for ovulation rate, number of viable embryos and embryo survival (Bidanel et al., 2008).

\section{Associations With Fertility Traits}

A total of 192 boars were assumed to be randomly mated with 3460 sows with unknown genotypes to generate 5340 litters (dataset 1). Nineteen haplotypes were significant for at least one fertility trait (Tables 2, 3). Haplotypes on SSC 3 at $87.1-87.5 \mathrm{Mb}$, SSC 8 at $145.1-145.5 \mathrm{Mb}$, and SSC 9 at 19.0-19.4 Mb affected both TNB $(P<0.05)$ and either BA or L24 $(P<0.10)$. In each case, litters sired by carrier boars had approximately 0.3 fewer piglets than those sired by boars that were not carriers of the recessive haplotype. The two overlapping haplotypes on SSC 8 spanning the interval from 144.9-145.5 both affected TNB. Haggman and Uimari (2017) detected one recessive haplotype at 107.0 - 113.3 Mb on SSC8 in Finnish Yorkshire which showed a significant effect on number of stillborn piglets in both first and later parities. Three distinct haplotypes on SSC9 affected at least one trait.

Several additional loci were detected as having effects on one of the fertility traits. An additional haplotype on SSC 8 at 73.3-73.7 Mb was associated with L24 with carrier boars siring litters with 0.24 fewer piglets that were alive at $24 \mathrm{~h}$ postpartum. Three haplotypes on SSC 4 at approximately $28 \mathrm{Mb}$ were each associated with the number of MM. Two distinct haplotypes, in addition to the locus described above, at 126.9127.3 and 15.3-15.7 $\mathrm{Mb}$ affected numbers of MM, with the latter haplotype also affecting the number of piglets L24. Two adjacent haplotypes on SSC 1 at approximately $82 \mathrm{Mb}$ were found to affect TNB or SB. Haplotypes on SSC12 at 35.8-36.2 and 50.1-50.5 Mb effected L24 and TNB, respectively. A QTL at approximately $31 \mathrm{Mb}$ was previously reported for number of embryos (Bidanel et al., 2008). Further downstream at 126.9127.3 $\mathrm{Mb}$ there was another haplotype that affected the number of MM.

Previously, two regions on SSC 1 at approximately 137 and $187 \mathrm{Mb}$ were found to have significant effect on TNB in Large White (Howard et al., 2017). Eight haplotypes whose homozygotes for their recessive alleles were missing in the present study support the result of Howard et al. (2017). However, effects on the fertility phenotypes were not detected here (Supplementary Table S1).

Fifty litters were generated from matings between boars and sows with known genotypes (dataset 2). In this smaller dataset, four haplotypes affected $(P<0.10)$ at least one fertility trait (Table 4). The haplotypes on SSC 8 at $145.1-145.5 \mathrm{Mb}$ and on SSC 9 at 19-19.4 Mb that were identified above again affected the numbers TNB and L24, respectively. In addition, the overlapping haplotypes located on SSC 17 at 28.7-29.1 and 28.9-29.3 Mb were significant for the three litter size traits. For the latter haplotype, litters from non-carrier mated with non-carrier had $1.96,2.13$, and 2.32 more piglets born, born live and alive at
$24 \mathrm{~h}$ post-partum, respectively, than litters from the mating of carriers.

\section{Candidate Gene Identification}

A total of 95 known genes were identified around the detected haplotype blocks that were found associated with at least one of the fertility traits. Seventeen of them (Table 5) are involved in the functional pathways of fertility, disease or organ development, which might be related to early embryonic mortality or life survival. Their phenotypic effects, primarily drawn for studies of model organisms, are briefly described below. In previous studies, approaches that are similar to those used to obtain the results described above were also used to identify candidate loci that are essential for life in swine (Derks et al., 2017; Haggman and Uimari, 2017; Howard et al., 2017). However, in general, there was relatively little commonality of QTL across studies. To date, the populations explored have been independent and largely distinct from each other.

x CYP26B1 (Cytochrome P450 Family 26 Subfamily B Member 1) close to haplotype 3 on SSC3 is expressed in the testis of embryonic mice and plays a central role in germ cell development, but not in ovarian tissue of likeaged female embryos (Koubova et al., 2006; Kipp et al., 2011). In three CYP26B1 mutant carrier $\mathrm{x}$ carrier families, in utero mortality of three individuals was observed in the first family, one patient in a second survived to 5 months of age, and in the third family an affected individual survived beyond infancy (Morton et al., 2016). PCF11 (cleavage complex II protein) is within haplotype 13 on SSC9. The mutations in PCF11 exhibit recessive lethality in Drosophila (Xie and Birchler, 2012). Haplotype 19 on SCC16 contains several important genes. TLX3 (T Cell Leukemia Homeobox 3) is related to the pathways of sudden infant death syndrome (SIDS) susceptibility and transcriptional misregulation in cancer (Weese-Mayer et al., 2004). The subunit encoded by GABRP (Gamma-Aminobutyric Acid Type A Receptor Pi Subunit) is expressed in several non-neuronal tissues including the uterus and ovaries (Kim et al., 2015). The presence of this subunit alters the sensitivity of recombinant receptors to modulatory agents such as pregnanolone. In the uterus, the function of the receptor appears to be related to tissue contractility.

Several genes within or around the significant haplotypes are involved in spermiogenesis related pathways which may affect prenatal survival. Haplotype 16 around 35.8 - $36.3 \mathrm{Mb}$ on SSC12 is relatively gene rich. TEX14 (Testis Expressed 14, Intercellular Bridge Forming Factor) and SEPT4 (Septin 4) showed significant effect on spermatogenesis and male fertility. Diseases associated with TEX14 include Spermatogenic Failure 23. Formation of intercellular bridges in the testis of mice requires expression of TEX14 (Greenbaum et al., 2006). These intercellular bridges are evolutionarily conserved structures that connect differentiating germ cells and are required for spermatogenesis and male fertility. SEPT4 is probably required for the structural integrity and motility of the sperm tail during postmeiotic differentiation (Kuo et al., 2015). SEPT4-null mice 
TABLE 4 | Effects of mating type (non-carrier $\times$ non-carrier $=00$, non-carrier $\times$ carrier $=01$, carrier $\times$ carrier $=11$ ) on TNB, BA and L24 as expressed in litters of purebred Duroc pigs.

\begin{tabular}{|c|c|c|c|c|c|c|c|}
\hline \multirow[t]{2}{*}{ Haplotype } & \multirow[t]{2}{*}{ SSC } & \multirow[t]{2}{*}{ Locus (Mb) } & \multirow[t]{2}{*}{ No. ${ }^{1}$} & \multicolumn{4}{|c|}{ Mating type } \\
\hline & & & & Trait $^{2}$ & 00 & 01 & 11 \\
\hline 11 & 8 & $145.1-145.5$ & $20 / 19 / 11$ & TNB & $11.71 \pm 0.54$ & $9.84 \pm 0.54$ & $10.14 \pm 0.72$ \\
\hline 13 & 9 & $19-19.4$ & $15 / 24 / 11$ & L24 & $10.59 \pm 0.70$ & $9.83 \pm 0.57$ & $8.57 \pm 0.74$ \\
\hline \multirow[t]{2}{*}{20} & 17 & $28.7-29.1$ & $27 / 20 / 3$ & $\mathrm{BA}$ & $10.12 \pm 0.43$ & $9.86 \pm 0.49$ & $7.19 \pm 1.26$ \\
\hline & & & & L24 & $10.09 \pm 0.42$ & $9.65 \pm 0.48$ & $7.18 \pm 1.23$ \\
\hline \multirow[t]{3}{*}{21} & 17 & 28.9-29.3 & $17 / 20 / 13$ & TNB & $11.59 \pm 0.56$ & $10.51 \pm 0.52$ & $9.63 \pm 0.65$ \\
\hline & & & & $\mathrm{BA}$ & $10.87 \pm 0.52$ & $9.65 \pm 0.47$ & $8.74 \pm 0.59$ \\
\hline & & & & L24 & $10.88 \pm 0.49$ & $9.50 \pm 0.45$ & $8.56 \pm 0.56$ \\
\hline
\end{tabular}

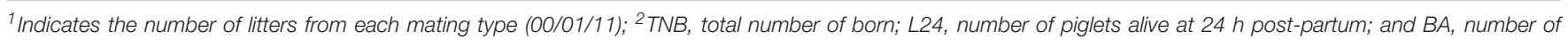
piglets born live.

have defects in caspase-mediated elimination of bulk cytoplasm during spermiogenesis and thus afflicted males are sterile but survive (Kissel et al., 2005; García-Fernández et al., 2010). HSF5 (Heat Shock Transcription Factor 5) may act as a transcriptional factor. Testes of adult HSF5-/- male zebrafish have relatively abundant spermatocytes and very few spermatozoa resulting from miss-regulation of the cell cycle and meiosis (Orbán et al., 2018). GTSF1 (Gametocyte Specific Factor 1) within haplotype 8 on SSC5 is required for spermatogenesis and is involved in the suppression of retrotransposon transcription in male germ cells.

A few genes in Haplotype 14 and Haplotype 17 are involved in tumor necrosis, e.g., FASLG (Fas Ligand), TNFSF4 (TNF Superfamily Member 4) and TNFSF18 (TNF Superfamily Member 18), or tumor suppression, e.g., RNF43 (Ring Finger Protein 43) and PCSK2 (Proprotein Convertase Subtilisin/Kexin Type 2). These genes are involved in important signaling pathways that are essential for immune system regulation, organ development, tumor development and progression. Mutations in these genes may affect essential functions resulting in more stillborn piglets. The protein encoded by FGF18 (Fibroblast Growth Factor 18) is a member of the fibroblast growth factor (FGF) family which are involved in a variety of biological processes including embryonic development (Usui et al., 2004; Hung et al., 2016). A few genes associated with disease were also found in this study. Both SCD5 (StearoylCoA Desaturase 5) and ENOPH1 (Enolase-Phosphatase 1) within Haplotypes 10-11 on SSC8 are related to the disease of Chromosome 4Q21 Deletion Syndrome. Mouse pups that do not express SCD5 are stillborn, with reduced growth rate in utero (Krakowiak et al., 2003). Diseases associated with ITGB1 (Integrin Subunit Beta 1) include Gallbladder Cancer and Breast Cancer. Integrin family members are membrane receptors involved in a variety of processes including embryogenesis, hemostasis, tissue repair, immune response and metastatic diffusion of tumor cells.

TABLE 5 | Candidate genes occurring within $400 \mathrm{~Kb}$ flanking of a haplotype for which the minor allele homozygous genotype was not observed and that had an effect on at least one of the fertility traits.

\begin{tabular}{|c|c|c|c|c|c|c|c|}
\hline Haplotype & ssc & Locus (Mb) & Gene name & Gene Start (bp) & Gene End (bp) & Distance (Kb) & Location \\
\hline 8 & 5 & $20.1-20.5$ & GTSF1 & $20,494,143$ & $20,504,958$ & 0 & Within \\
\hline & & & ENOPH1 & $145,145,811$ & $145,172,579$ & 0 & Within \\
\hline 13 & 9 & $19-19.4$ & PCF11 & $19,211,763$ & $19,238,971$ & 0 & Within \\
\hline \multirow[t]{2}{*}{14} & 9 & 126.9-127.3 & FASLG & $126,641,117$ & $126,647,827$ & -259 & Up-stream \\
\hline & & & TNFSF18 & $127,140,871$ & $127,151,423$ & 0 & Within \\
\hline 15 & 10 & $61.8-62.2$ & ITGB1 & $61,450,695$ & $61,502,941$ & -297 & Up-stream \\
\hline \multirow[t]{4}{*}{16} & 12 & $35.8-36.2$ & TEX14 & $35,849,615$ & $36,000,403$ & 0 & Within \\
\hline & & & SEPT4 & $36,030,882$ & $36,043,170$ & 0 & Within \\
\hline & & & HSF5 & $36,070,712$ & $36,117,782$ & 0 & Within \\
\hline & & & RNF43 & $36,120,600$ & $36,181,669$ & 0 & Within \\
\hline $20-21$ & 17 & $28.7-29.3$ & PCSK2 & $29,095,048$ & $29,329,854$ & 0 & Within \\
\hline
\end{tabular}


For haplotype 4 on SSC3, no known gene was detected around this block. However, a few interesting genes (not shown in Table 5) around 1-3 $\mathrm{Mb}$ of this block were involved in some important pathways of reproduction or life. FSHR is the receptor for follicle stimulating hormone and functions in gonad development (Costagliola et al., 2002). Mutations in this gene cause ovarian dysgenesis type 1 and ovarian hyperstimulation syndrome (Uchida et al., 2013). EPCAM (Epithelial Cell Adhesion Molecule) plays a role in embryonic stem cell proliferation and differentiation. Mutations in CALM2 (Calmodulin 2) or another member of the calmodulin gene family seem to result in patients with severe forms of longQT syndrome (LQTS) who displayed life-threatening ventricular arrhythmias, a rare disorder thought to be the cause of a significant fraction of sudden cardiac deaths in young individuals, and together with delayed neurodevelopment and epilepsy (Nyegaard et al., 2012; Crotti et al., 2013; Makita et al., 2014).

\section{CONCLUSION}

These findings facilitate potentially increasing Duroc litter size through a marker-assisted breeding and mate selection designed to avoid producing homozygous zygotes. If these technologies can be successfully implemented, breeders should benefit from increased selection intensity as well as having an increased number of pigs to market from each litter. In addition, the results serve to validate previous findings as to loci which affect prenatal mortality and consequently litter size. The identified candidate genes may also lead to new insights for the biology of pig fertility.

\section{ETHICS STATEMENT}

Data and samples for genotyping were collected at Genesus Inc. Research and Development Facilities (Genesus Inc., Oakville, $\mathrm{MB}$, Canada) as part of routine commercial operation prior to the research. The proposed work was reviewed by the University of Alberta Animal Care and Use Committee. No other specific

\section{REFERENCES}

Bidanel, J. P., Rosendo, A., Iannuccelli, N., Riquet, J., Gilbert, H., Caritez, J. C., et al. (2008). Detection of quantitative trait loci for teat number and female reproductive traits in Meishan X Large White F2 pigs. Animal 2, 813-820. doi: $10.1017 /$ S1751731108002097

Bolet, G. (1986). Timing and Extent of Embryonic Mortality in Pigs Sheep and Goats: Genetic Variability in Embryonic mortality in farm animals. Berlin: Springer, $12-43$.

Christianson, W. T. (1992). Stillbirths, mummies, abortions, and early embryonic death. Vet. Clin. North Am. Food Anim. Pract. 8, 623-639. doi: 10.1016/S07490720(15)30708-8

Costagliola, S., Panneels, V., Bonomi, M., Koch, J., Many, M. C., Smits, G., et al. (2002). Tyrosine sulfation is required for agonist recognition by glycoprotein hormone receptors. EMBO J. 21, 504-513. doi: 10.1093/emboj/21.4.504

Crotti, L., Johnson, C. N., Graf, E., De Ferrari, G. M., Cuneo, B. F., Ovadia, M., et al. (2013). Calmodulin mutations associated with recurrent cardiac arrest in infants. Circulation 127, 1009-1017. doi: 10.1161/CIRCULATIONAHA.112. 001216 permissions were required for the work, since the animals were cared for according to the Canadian Quality Assurance Program, which includes attention to animal health and well-being and is in line with the Canadian Council on Animal Care guidelines.

\section{AUTHOR CONTRIBUTIONS}

GP, MM, and MD conceived and designed the experiments. CZ performed the statistical analysis. GP and MM provided valuable advice to refine the statistical analyses. RK contributed materials and application advice. CZ, GP, and MM were major contributors in writing the manuscript. All authors read and approved the final manuscript.

\section{FUNDING}

Alberta Livestock and Meat Agency, Genome Alberta, and in kind support from the industry partner Genesus Inc.

\section{ACKNOWLEDGMENTS}

We are thanks to all the staff and technicians who collected the samples and data.

\section{SUPPLEMENTARY MATERIAL}

The Supplementary Material for this article can be found online at: https://www.frontiersin.org/articles/10.3389/fgene. 2018.00655/full\#supplementary-material

TABLE S1 | Location and minor allele frequency for haplotypes which were expected to have two homozygous genotypes, but with only one found in the data and which did not have a significant effect on any of the fertility phenotypes. ${ }^{1}$ Number of SNPs in the haplotype blocks; ${ }^{2}$ Haplotype index; ${ }^{3}$ number of individuals that were expected to be homozygous for the minor allele; ${ }^{4}$ Bonferroni corrected $P$-value.

Delaneau, O., Marchini, J., and Genomes Project Consortium (2014). Integrating sequence and array data to create an improved 1000 genomes project haplotype reference panel. Nat. Commun. 5:3934. doi: 10.1038/ncomms4934

Delaneau, O., Marchini, J., and Zagury, J. F. (2012). A linear complexity phasing method for thousands of genomes. Nat. Methods 9, 179-181. doi: 10.1038/ Nmeth.1785

Derks, M. F. L., Megens, H. J., Bosse, M., Lopes, M. S., Harlizius, B., and Groenen, M. A. M. (2017). A systematic survey to identify lethal recessive variation in highly managed pig populations. BMC Genomics 18:858. doi: 10.1186/s12864017-4278-1

García-Fernández, M., Kissel, H., Brown, S., Gorenc, T., Schile, A. J., Rafii, S., et al. (2010). Sept4/ARTS is required for stem cell apoptosis and tumor suppression. Genes Dev. 24, 2282-2293. doi: 10.1101/gad.1970110

Geisert, R., and Schmitt, R. (2002). Early embryonic survival in the pig: can it be improved? 1. J. Anim. Sci. 80, E54-E65.

Gilmour, A., Gogel, B., Cullis, B., Thompson, R., Butler, D., Cherry, M., et al. (2008). ASReml User Guide Release 3.0. Hemel Hempstead: VSN Int Ltd.

Greenbaum, M. P., Yan, W., Wu, M. H., Lin, Y. N., Agno, J. E., Sharma, M., et al. (2006). TEX14 is essential for intercellular bridges and fertility in male 
mice. Proc. Natl. Acad. Sci. U.S.A. 103, 4982-4987. doi: 10.1073/pnas.05051 23103

Grossi, D. A., Jafarikia, M., Brito, L. F., Buzanskas, M. E., Sargolzaei, M., and Schenkel, F. S. (2017). Genetic diversity, extent of linkage disequilibrium and persistence of gametic phase in Canadian pigs. BMC Genet. 18:6. doi: 10.1186/ s12863-017-0473-y

Hadfield, J. D. (2010). MCMC methods for multi-response generalized linear mixed models: the MCMCglmm R package. J. Stat. Softw. 33, 1-22. doi: 10. 18637/jss.v033.i02

Haggman, J., and Uimari, P. (2017). Novel harmful recessive haplotypes for reproductive traits in pigs. J. Anim. Breed. Genet. 134, 129-135. doi: 10.1111/ jbg. 12240

Hoff, J. L., Decker, J. E., Schnabel, R. D., and Taylor, J. F. (2017). Candidate lethal haplotypes and causal mutations in Angus cattle. BMC Genomics 18:799. doi: 10.1186/s12864-017-4196-2

Howard, D. M., Pong-Wong, R., Knap, P. W., and Woolliams, J. A. (2017). Use of haplotypes to identify regions harbouring lethal recessive variants in pigs. Genet. Sel. Evol. 49:57. doi: 10.1186/s12711-017-0332-3

Hung, I. H., Schoenwolf, G. C., Lewandoski, M., and Ornitz, D. M. (2016). A combined series of Fgf9 and Fgf18 mutant alleles identifies unique and redundant roles in skeletal development. Dev. Biol. 411, 72-84. doi: 10.1016/ j.ydbio.2016.01.008

Johnson, R. K. (1981). Crossbreeding in swine: experimental results 1. J. Anim. Sci. 52, 906-923. doi: 10.2527/jas1981.524906x

Kim, H. S., Jin, E. H., Mo, J. S., Shim, H., Lee, S. S., and Chae, S. C. (2015). The association of the GABRP polymorphisms with systemic lupus erythematosus. J. Immunol. Res. 2015:602154. doi: 10.1155/2015/602154

Kipp, J. L., Golebiowski, A., Rodriguez, G., Demczuk, M., Kilen, S. M., and Mayo, K. E. (2011). Gene expression profiling reveals Cyp26b1 to be an activin regulated gene involved in ovarian granulosa cell proliferation. Endocrinology 152, 303-312. doi: 10.1210/en.2010-0749

Kissel, H., Georgescu, M. M., Larisch, S., Manova, K., Hunnicutt, G. R., and Steller, H. (2005). The Sept4 septin locus is required for sperm terminal differentiation in mice. Dev. Cell 8, 353-364. doi: 10.1016/j.devcel.2005.01.021

Koubova, J., Menke, D. B., Zhou, Q., Capel, B., Griswold, M. D., and Page, D. C. (2006). Retinoic acid regulates sex-specific timing of meiotic initiation in mice. Proc. Natl. Acad. Sci. U.S.A. 103, 2474-2479. doi: 10.1073/pnas.0510813103

Krakowiak, P. A., Wassif, C. A., Kratz, L., Cozma, D., Kovarova, M., Harris, G., et al. (2003). Lathosterolosis: an inborn error of human and murine cholesterol synthesis due to lathosterol 5-desaturase deficiency. Hum. Mol. Genet. 12, 1631-1641. doi: 10.1093/hmg/ddg172

Kuo, Y. C., Shen, Y. R., Chen, H. I., Lin, Y. H., Wang, Y. Y., Chen, Y. R., et al. (2015). SEPT12 orchestrates the formation of mammalian sperm annulus by organizing core octameric complexes with other SEPT proteins. J. Cell Sci. 128, 923-934. doi: $10.1242 /$ jcs. 158998

Lonergan, S. M., Huff-Lonergan, E., Rowe, L. J., Kuhlers, D. L., and Jungst, S. B. (2001). Selection for lean growth efficiency in Duroc pigs influences pork quality. J. Anim. Sci. 79, 2075-2085. doi: 10.2527/2001.7982075x

Makita, N., Yagihara, N., Crotti, L., Johnson, C. N., Beckmann, B. M., Roh, M. S., et al. (2014). Novel calmodulin mutations associated with congenital arrhythmia susceptibility. Circ. Genom. Precis. Med. 7, 466-474. doi: 10.1161/ CIRCGENETICS.113.000459

Morton, J. E., Frentz, S., Morgan, T., Sutherland-Smith, A. J., and Robertson, S. P. (2016). Biallelic mutations in CYP26B1: a differential diagnosis for pfeiffer and antley-bixler syndromes. Am. J. Med. Genet. 170, 2706-2710. doi: 10.1002/ajmg. a.37804

Noguera, J. L., Varona, L., Babot, D., and Estany, J. (2002). Multivariate analysis of litter size for multiple parities with production traits in pigs: II. Response to selection for litter size and correlated response to production traits. J. Anim. Sci. 80, 2548-2555. doi: 10.2527/2002.80102548x

Nyegaard, M., Overgaard, M. T., Sondergaard, M. T., Vranas, M., Behr, E. R., Hildebrandt, L. L., et al. (2012). Mutations in calmodulin cause ventricular tachycardia and sudden cardiac death. Am. J. Hum. Genet. 91, 703-712. doi: 10.1016/j.ajhg.2012.08.015

Orbán, L., Saju, J. M., Hossain, M. S., Liew, W. C., Pradhan, A., Thevasagayam, N. M., et al. (2018). Heat shock factor 5 is conserved in vertebrates and essential for spermatogenesis in zebrafish. bioRxiv [Preprint]. doi: 10.1101/254615
Sahana, G., Nielsen, U. S., Aamand, G. P., Lund, M. S., and Guldbrandtsen, B. (2013). Novel harmful recessive haplotypes identified for fertility traits in Nordic Holstein cattle. PLoS One 8:e82909. doi: 10.1371/journal.pone.0082909

Sonderman, J. P., and Luebbe, J. J. (2008). Semen production and fertility issues related to differences in genetic lines of boars. Theriogenology 70, 1380-1383. doi: 10.1016/j.theriogenology.2008.08.009

Sorensen, D., Vernersen, A., and Andersen, S. (2000). Bayesian analysis of response to selection: a case study using litter size in danish yorkshire pigs. Genetics 156, 283-295.

Tortereau, F., Servin, B., Frantz, L., Megens, H. J., Milan, D., Rohrer, G., et al. (2012). A high density recombination map of the pig reveals a correlation between sex-specific recombination and GC content. BMC Genomics 13:586. doi: 10.1186/1471-2164-13-586

Uchida, S., Uchida, H., Maruyama, T., Kajitani, T., Oda, H., Miyazaki, K., et al. (2013). Molecular analysis of a mutated FSH receptor detected in a patient with spontaneous ovarian hyperstimulation syndrome. PLoS One 8:e75478. doi: 10.1371/journal.pone.0075478

Usui, H., Shibayama, M., Ohbayashi, N., Konishi, M., Takada, S., and Itoh, N. (2004). Fgf18 is required for embryonic lung alveolar development. Biochem. Biophys. Res. Commun. 322, 887-892. doi: 10.1016/j.bbrc.2004.07.198

Utsunomiya, Y. T., Milanesi, M., Utsunomiya, A. T., Ajmone-Marsan, P., and Garcia, J. F. (2016). GHap: an R package for genome-wide haplotyping. Bioinformatics 32, 2861-2862. doi: 10.1093/bioinformatics/btw356

VanRaden, P. M., Olson, K. M., Null, D. J., and Hutchison, J. L. (2011). Harmful recessive effects on fertility detected by absence of homozygous haplotypes. J. Dairy Sci. 94, 6153-6161. doi: 10.3168/jds.2011-4624

Veroneze, R., Lopes, P. S., Guimaraes, S. E., Silva, F. F., Lopes, M. S., Harlizius, B., et al. (2013). Linkage disequilibrium and haplotype block structure in six commercial pig lines. J. Anim. Sci. 91, 3493-3501. doi: 10.2527/jas. 2012-6052

Weese-Mayer, D. E., Berry-Kravis, E. M., Zhou, L., Maher, B. S., Curran, M. E., Silvestri, J. M., et al. (2004). Sudden infant death syndrome: case-control frequency differences at genes pertinent to early autonomic nervous system embryologic development. Pediatr. Res. 56, 391-395. doi: 10.1203/01.PDR. $0000136285.91048 .4 \mathrm{~A}$

Xie, W. W., and Birchler, J. A. (2012). Identification of inverse regulator-a (Inr-a) as synonymous with pre-mrna cleavage complex II protein (pcf11) in drosophila. G3 2, 701-706. doi: 10.1534/g3.112.002071

Young, L. D., Johnson, R. K., and Omtvedt, I. T. (1976). Reproductive performance of swine bred to produce purebred and two-breed cross litters. J. Anim. Sci. 42, 1133-1149. doi: 10.2527/jas1976.4251133x

Zhang, C., Kemp, R. A., Stothard, P., Wang, Z., Boddicker, N., Krivushin, K., et al. (2018). Genomic evaluation of feed efficiency component traits in Duroc pigs using $80 \mathrm{~K}, 650 \mathrm{~K}$ and whole-genome sequence variants. Genet. Sel. Evol. 50:14. doi: 10.1186/s12711-018-0387-9

Zhang, H., Aalhus, J. L., Gariepy, C., Uttaro, B., Lopez-Campos, O., Prieto, N., et al. (2016). Effects of pork differentiation strategies in Canada on pig performance and carcass characteristics. Can. J. Anim. Sci. 96, 512-523. doi: 10.1139/cjas2015-0197

Conflict of Interest Statement: RK is employed by Genesus Inc. who provided data and genotypes as an in kind contribution to the research. RK was involved in the sample and data collection and contributed to the paper by advising on the potential application of the results. The potential conflict of interest did not interfere with the outcome of this paper.

The remaining authors declare that the research was conducted in the absence of any commercial or financial relationships that could be construed as a potential conflict of interest.

Copyright (c) 2018 Zhang, MacNeil, Kemp, Dyck and Plastow. This is an open-access article distributed under the terms of the Creative Commons Attribution License (CC BY). The use, distribution or reproduction in other forums is permitted, provided the original author(s) and the copyright owner(s) are credited and that the original publication in this journal is cited, in accordance with accepted academic practice. No use, distribution or reproduction is permitted which does not comply with these terms. 